\title{
Expression profile analysis reveals putative prostate cancer-related microRNAs
}

\author{
H. Song ${ }^{1 *}$, Y. Liu ${ }^{2 *}$, J. Pan $^{3}$ and S.T. Zhao ${ }^{1}$ \\ ${ }^{1}$ Department of Urology, The Second Hospital of Shandong University, \\ Jinan, China \\ ${ }^{2}$ Department of Urology, General Hospital of Jinan Military Command, \\ Jinan, China \\ ${ }^{3}$ Jinan Municipal Center for Disease Control and Prevention, Jinan, China \\ *These authors contributed equally to this study. \\ Corresponding author: S.T. Zhao \\ E-mail: zhaoshengtianzhao@hotmail.com
}

Genet. Mol. Res. 12 (4): 4934-4943 (2013)

Received March 7, 2013

Accepted August 30, 2013

Published October 24, 2013

DOI http://dx.doi.org/10.4238/2013.October.24.4

\begin{abstract}
Annotation of prostate cancer (PC) genomes provides a foundation for discoveries that can improve the understanding and treatment of the disease. Therefore, in the present study, we used the Student $t$-test to identify differentially expressed PC-related mRNAs and microRNAs (miRNAs). Then, we performed interrelated mapping of miRNA target genes between abnormally expressed mRNAs and miRNAs, and explored mRNA-target miRNA interrelated pairs to explain the biological functions of miRNA during the progression of PC, thus revealing the occurrence of miRNA-mediated PC. After Gene Set Functional Similarity analysis, we obtained 20 abnormal PC-related candidate miRNAs, including hsa-miR-26a, hsa-miR-152, hsa-miR19a, hsa-miR-30c, hsa-miR-19b, and hsa-miR-146b-5p, among others. These results suggest that it may be possible to predict the clinical behavior of prostate cancer based on gene expression analysis.
\end{abstract}

Key words: miRNA; mRNA; Prostate cancer; Target gene; Gene set; Functional similarity 


\section{INTRODUCTION}

Prostate cancer (PC) is a form of cancer that develops in the prostate gland in the male reproductive system. Although there are cases of aggressive prostate cancers (Hessels et al., 2003), most are slow growing (Marks et al., 2007). Many factors have been implicated in the development of prostate cancer, including genetics and diet (Siegel et al., 2011). The presence of PC may be indicated by symptoms, a physical examination, prostate-specific antigens (PSA), or biopsy (Baade et al., 2009). PSA testing increases cancer detection but does not decrease PC-associated mortality rates (Djulbegovic et al., 2010).

MicroRNA (miRNA) expression profile studies have been conducted to identify cancer-specific miRNA signatures (Iorio et al., 2005; Akao et al., 2006; Cummins et al., 2006). miRNAs are small, endogenously expressed non-coding RNAs that negatively regulate the expression of protein-coding genes at the translational level (Lim et al., 2005) either by triggering degradation or by preventing translation of the target mRNAs (He and Hannon, 2004). Upon their discovery, miRNAs were shown to control fundamental cellular processes, such as cell differentiation and developmental timing (Lee et al., 1993; Reinhart et al., 2000). This suggested that miRNA aberrations could be involved in various human diseases, including cancer, which has since been confirmed with accumulating evidence of aberrant expression miRNA profiles. These have been identified in various tumor types, showing that different sets of miRNAs are usually deregulated in different cancers (Yanaihara et al., 2006; Tsang and Kwok, 2008).

Thus, genomic methodologies have been successfully used to discover consistent gene expression patterns associated with a given histological or clinical phenotype (Golub et al., 1999; Perou et al., 2000; van 't Veer et al., 2002). In the present study, based on RNA expression data, we obtained 2160 differentially expressed mRNAs and 71 differentially expressed miRNAs associated with PC. Furthermore, we selected 27 biological processes and 4 biological pathways using functional and pathway-enrichment analyses to shed light on the mechanism of miRNAs in PC. These results predicted that hsa-miR-26a was significantly related to eight known PC-related miRNAs, hsa-miR-152 was related to seven, and hsa-miR19a, hsa-miR-30c, hsa-miR-19b, and hsa-miR-146b-5p were related to six known PC-related miRNAs, which could be used as PC markers.

\section{MATERIAL AND METHODS}

\section{Expression profile data source of PC-related mRNAs and miRNAs}

PC-related mRNA expression profile data of GSE21034 and PC-related miRNA expression profile data of GSE21036 (Taylor et al., 2010) were obtained from a public functional genomic data repository, Gene Expression Omnibus (http://www.ncbi.nlm.nih.gov/geo/) that is based on the Affymetrix Human Exon 1.0 ST Array. The expression profile of mRNAs contained 150 PC samples and 20 control samples, while the expression profile of miRNAs contained 113 PC samples and 28 control samples.

Ninety-one PC-related miRNAs were obtained from the Human miRNA and Disease Database (http://202.38.126.151/hmdd/mirna/md/), including nine abnormally expressed 
miRNAs (hsa-miR-17, hsa-miR-20a, hsa-miR-106b, hsa-miR-93, hsa-miR-143, hsa-miR133b, hsa-miR-99a, hsa-miR-647, and hsa-miR-130a). Putative target genes were selected from the miRBase database using the target gene prediction algorithm, miRanda.

\section{Differential expression analysis}

In order to recognize the differentially expressed mRNAs and miRNAs between $\mathrm{PC}$ and control samples, we used the Student $t$-test to screen expression data of PC-related mRNAs and miRNAs. These abnormal factors may mediate the pathophysiological mechanism associated with PC. The P value was corrected using the false-discovery rate (FDR), in which the threshold was set to 0.05 .

\section{Function and pathway annotation}

In order to recognize the mechanism of miRNAs in the process of PC, we performed interrelated mapping of miRNA target genes between abnormally expressed mRNAs and miRNAs, and obtained mRNA-target miRNA interrelated pairs. The function and pathway annotation enabled identification of the biological functions of miRNAs during the progression of PC, and thus revealed the occurrence of miRNA-mediated PC. We used the DAVID program to perform Gene Ontology (GO) and KEGG-enrichment analysis, and the threshold of the $\mathrm{P}$ value for significance after FDR multiple-test correction was set to 0.01 .

\section{Analysis of significance of gene set functional similarity}

Gene Set Functional Similarity (GSFS) is a novel method for quantifying gene set functional associations based on GO using a semantic similarity algorithm (http://www. geneontology.org/GO.tools_by_type.functional_similarity.shtml). This method can also score and perform a significance analysis of GSFS. The significance level was set to 0.01 with 100 randomized trials.

\section{RESULTS}

\section{Experimental procedure introduction}

First, abnormal mRNAs and miRNAs were recognized within the expression profiles of PC-related mRNAs and miRNAs. Combining these with miRanda target gene prediction algorithms, we obtained abnormal miRNA-abnormal target mRNA interrelated pairs, and constructed the transcriptional regulatory network of disorders. Next, in order to reveal the biological function of the transcriptional regulatory network of disorders, we predicted the function of the contained miRNAs through enrichment of biological processes and pathways, based on their similarity to miRNA target genes. Finally, we predicted the potential PC-related miRNA genes (Figure 1). 


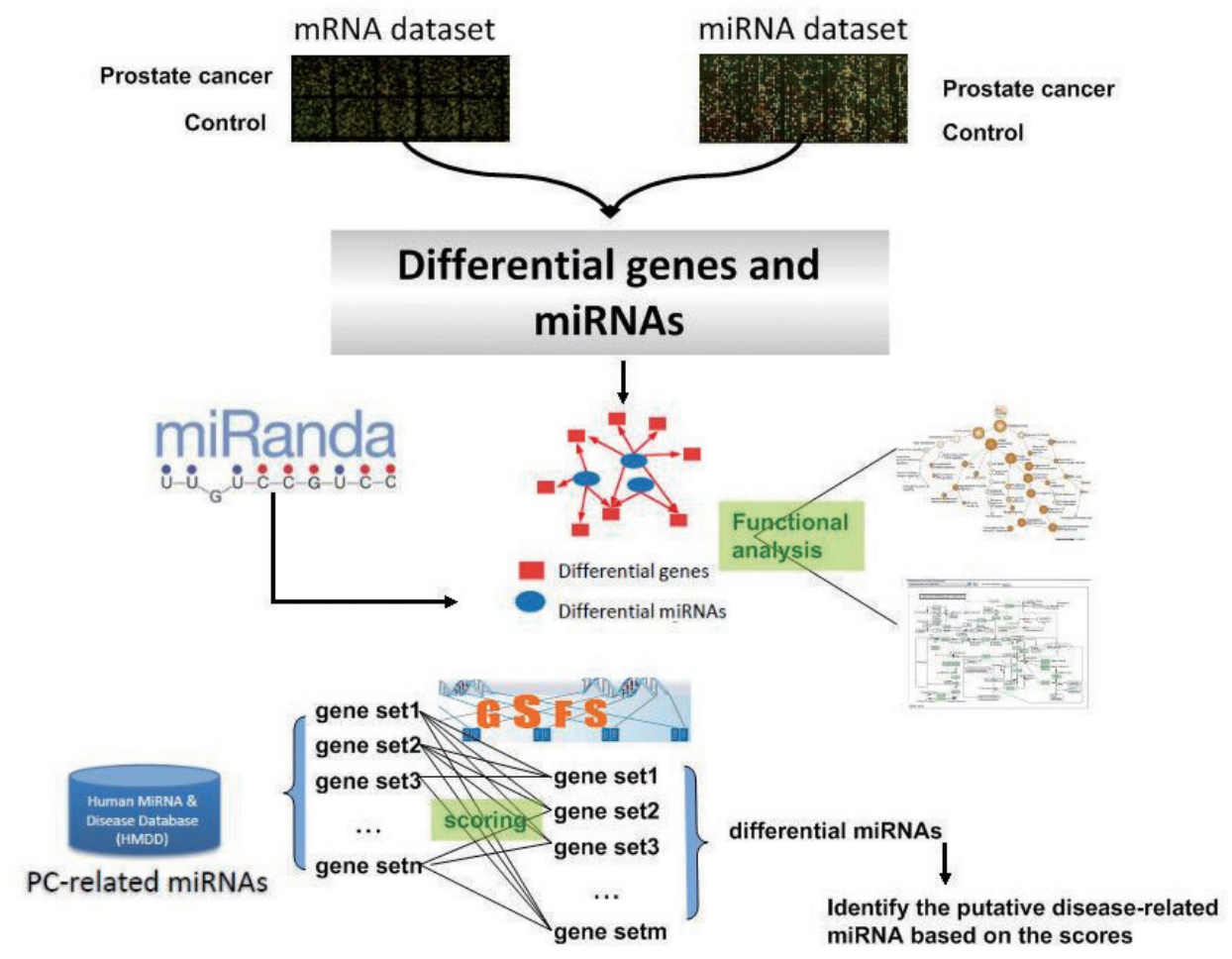

Figure 1. Scheme for the prediction of putative prostate cancer (PC)-related miRNAs based on functional associations.

\section{Differential expression profile analysis}

RNA expression data were compared between PC samples and normal samples with the Student $t$-test to determine differential expression patterns; the $\mathrm{P}$ value was corrected for multiple comparisons to a significance level of FDR $=0.01$. A total of 2160 differentially expressed mRNAs and 71 differentially expressed miRNAs were obtained.

\section{PC-related transcriptional regulatory network of disorders}

miRNA controls the translation of, or degrades, its target gene by binding to it. The miRBase database was used to obtain the miRanda algorithm for predicting conservative miRNA target genes. This revealed 74 abnormal target genes of miRNAs from 2160 mRNAs, and 8887 pairs of abnormal miRNA-mRNA pairs, including 28 abnormal miRNAs and 1701 abnormal mRNAs. Eight of the miRNAs were known disease miRNAs.

In order to detect the mechanism of miRNAs in PC, we selected 27 biological processes (Table 1) and 4 biological pathways (Table 2, Figures 2 and 3) using functional and pathway-enrichment analyses based on a $\mathrm{P}$ value of 0.01 . 
Table 1. Gene Ontology (GO) annotation of abnormal miRNA target genes.

\begin{tabular}{|c|c|c|}
\hline BPID & $P$ value & Term \\
\hline GO:0007242 & 4.03E-05 & Intracellular signaling cascade \\
\hline GO:0007264 & $1.23 \mathrm{E}-04$ & Small GTPase-mediated signal transduction \\
\hline GO:0042476 & $1.89 \mathrm{E}-04$ & Odontogenesis \\
\hline GO:0015031 & 8.13E-04 & Protein transport \\
\hline GO:0001894 & 0.001025 & Tissue homeostasis \\
\hline GO:0048729 & 0.001146 & Tissue morphogenesis \\
\hline GO:0009887 & 0.00147 & Organ morphogenesis \\
\hline GO:0060323 & 0.001592 & Head morphogenesis \\
\hline GO:0030834 & 0.001789 & Regulation of actin filament depolymerization \\
\hline GO:0035108 & 0.002499 & Limb morphogenesis \\
\hline GO:0000904 & 0.003011 & Cell morphogenesis involved in differentiation \\
\hline GO:0051347 & 0.004183 & Positive regulation of transferase activity \\
\hline GO:0001822 & 0.004309 & Kidney development \\
\hline GO:0009966 & 0.004876 & Regulation of signal transduction \\
\hline GO:0042733 & 0.004885 & Embryonic digit morphogenesis \\
\hline GO:0060325 & 0.005385 & Face morphogenesis \\
\hline GO:0001568 & 0.005779 & Blood vessel development \\
\hline GO:0045892 & 0.005968 & Negative regulation of transcription, DNA-dependent \\
\hline GO:0042475 & 0.006067 & Odontogenesis of dentine-containing tooth \\
\hline GO:0006464 & 0.006829 & Protein modification process \\
\hline GO:0008654 & 0.00803 & Phospholipid biosynthetic process \\
\hline GO:0051253 & 0.008084 & Negative regulation of RNA metabolic process \\
\hline GO:0000086 & 0.008131 & $\mathrm{G} 2 / \mathrm{M}$ transition of mitotic cell cycle \\
\hline GO:0001944 & 0.008341 & Vascular development \\
\hline GO:0043242 & 0.008548 & Negative regulation of protein complex disassembly \\
\hline GO:0030326 & 0.009073 & Embryonic limb morphogenesis \\
\hline GO:0035113 & 0.009073 & Embryonic appendage morphogenesis \\
\hline
\end{tabular}

BPID $=$ biological process identification.

Table 2. KEGG pathways annotation of abnormal miRNA target genes.

\begin{tabular}{llr}
\hline Pathway & MAPP name & Adjusted P \\
\hline Hsa 00564 & Glycerophospholipid metabolism & 0.001617 \\
Hsa 04512 & ECM-receptor interaction & 0.004807 \\
Hsa 00565 & Ether lipid metabolism & 0.008354 \\
Hsa 04070 & Phosphatidylinositol signaling system & 0.009637 \\
\hline
\end{tabular}

In differentially expressed genes, 16 miRNA target genes were enriched into extracellular matrix (ECM)-receptor interaction pathway (Figure 2), and 14 miRNA target genes were enriched into phosphatidylinositol-signaling pathway (Figure 3).

\section{Predicting potential PC-related miRNAs}

Because miRNAs use their target genes to achieve their regulatory functions, different miRNAs that are involved in target genes with similar regulatory functions may be associated. Based on this hypothesis, we used the functional interaction of abnormal target genes to predict the pathogenesis of abnormal miRNAs. First, using eight abnormal disease miRNAs for the seed based on the miRanda algorithm, we selected the target gene set of each disease miRNA. Then, using the disease miRNA target set and the abnormal miRNA set based on the GSFS algorithm to score the functional similarity of each gene set, which was randomly perturbed 100 times and set to a probability of 0.01 , we calculated the functional similarity score matrix between gene sets and the abnormal miRNA sequences. 


\section{ECM-RECEPTOR INTERACTION}
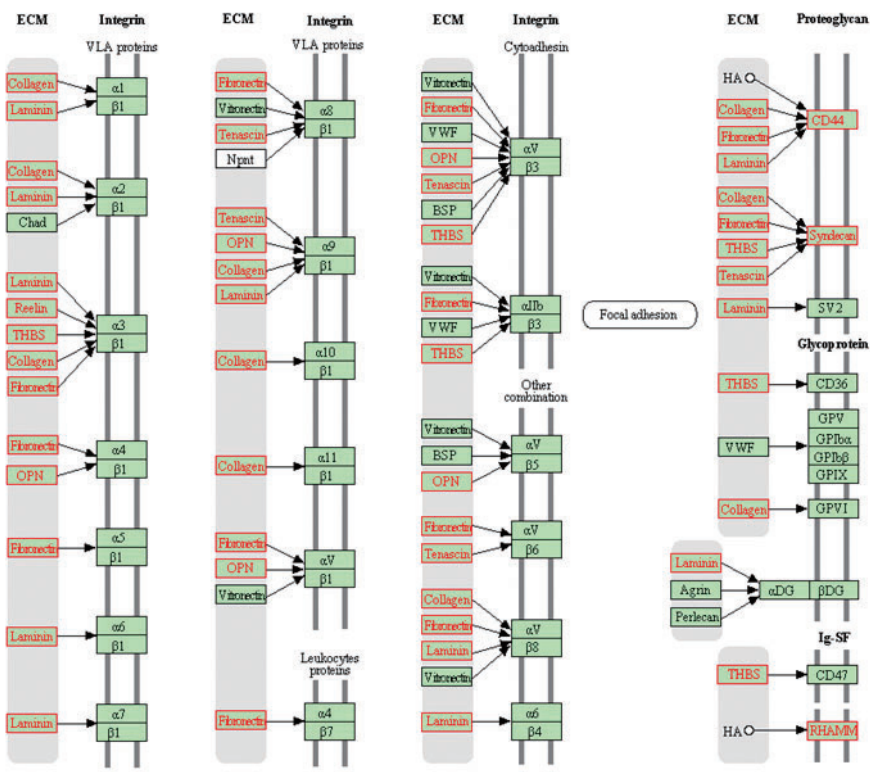

Figure 2. Abnormal target genes of differentially expressed miRNAs were significantly enriched in the extracellular matrix (ECM)-receptor interaction pathway. The $\mathrm{P}$ value calculated by the hypergeometric distribution was set to 0.01 . Overexpressed genes are shown in red.

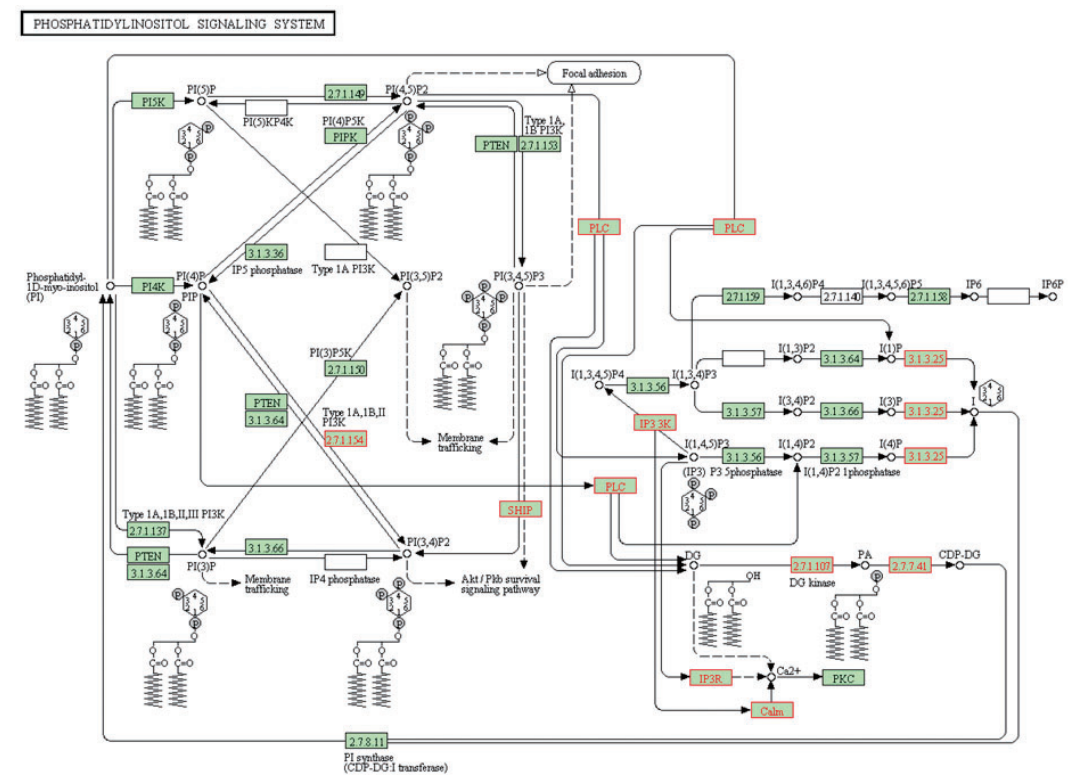

Figure 3. Abnormal target genes of differentially expressed miRNAs were significantly enriched in the phosphatidylinositol-signaling system pathway. The $\mathrm{P}$ value calculated by the hypergeometric distribution was set to 0.01 . Overexpressed genes are shown in red. 
The process used to identify PC-related candidate miRNAs was as follows. The score was calculated based on the similarity between the abnormal miRNA set and the eight disease miRNA set. If the function of an abnormal miRNA was more similar to the disease miRNAs, it was considered to be particularly relevant to PC. In other words, the higher the Simscore, the more likely the miRNA was considered to be involved in the pathogenesis of PC. As shown in Figure 4, of the 20 abnormal miRNAs, hsa-miR-26a and eight known PC-related miRNAs were significantly related, while hsa-miR-152 was related to seven, and hsa-miR-19a, hsamiR-30c, hsa-miR-19b, and hsa-miR-146b-5p were related to six known PC-related miRNAs.

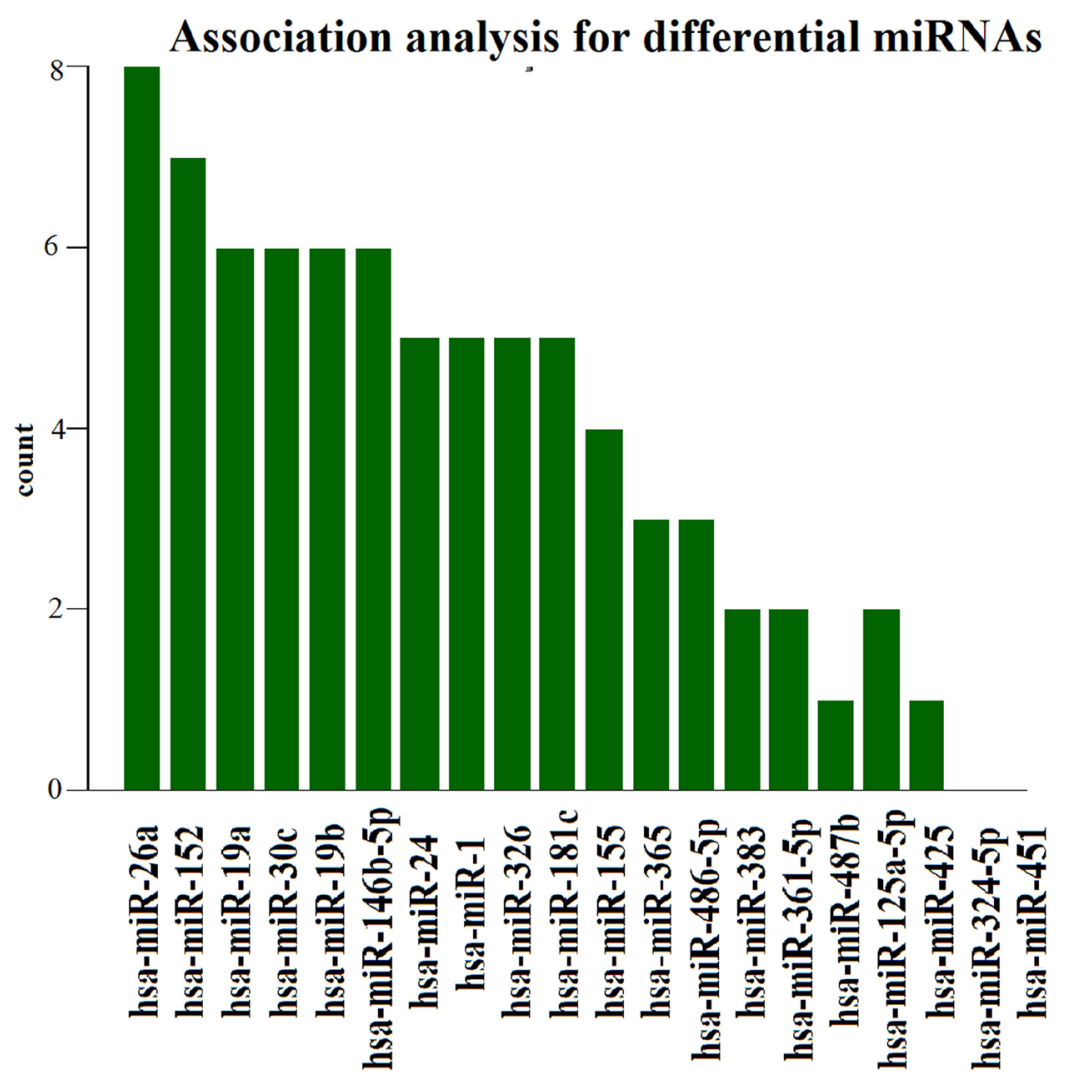

Figure 4. Prediction of putative miRNAs associated with prostate cancer based on functional similarity of target sets.

\section{DISCUSSION}

Annotation of PC genomes provides a foundation for discoveries that can improve the understanding and treatment of the disease. PC-related mRNA expression profile data of GSE21034 and PC-related miRNA expression profile data of GSE21036 were analyzed to identify the prostate cancer miRNA signature and to predict PC-related miRNAs. 
After finding differentially expressed mRNAs and miRNAs, we constructed a PCrelated transcriptional regulatory network of disorders. Using this network, we selected 27 biological processes (Table 1) and four biological pathways (Table 2) using functional and pathway-enrichment analyses. The 27 biological processes varied widely, including intracellular signaling cascades (Nishizuka, 1992), small GTPase-mediated signal transduction (Clark and Brugge, 1995), and the G2/M transition of the mitotic cell cycle (Abrieu et al., 1998), among others. Cell signaling has been most extensively studied in the context of human diseases and signaling between cells of a single organism. Some signaling transduction pathways can respond differently depending on the amount of signaling received by the cell. For instance, the hedgehog protein activates different genes depending on the amount of hedgehog protein present (Dinasarapu et al., 2011). GTPases are a large family of hydrolase enzymes that can bind and hydrolyze guanosine triphosphate (GTP). The GTP binding and hydrolysis take place in the highly conserved G domain common to all GTPases (Scheffzek and Ahmadian, 2005). The G2-M DNA damage checkpoint is an important cell cycle checkpoint in eukaryotic organisms ranging from yeast to mammals. This checkpoint ensures that cells do not initiate mitosis before they have a chance to repair any damaged DNA after replication. Cells that have a defective G2-M checkpoint enter mitosis before repairing their DNA, leading to death after cell division (Cuddihy and O'Connell, 2003).

Target gene selection and functional pathway-enrichment analysis revealed four biological pathways. Pathway annotation of target mRNAs regulated by miRNAs indicated that these genes were significantly enriched in extracellular matrix (ECM)-receptor interactions. Because of its diverse nature and composition, the ECM can serve many functions, such as providing support, segregating tissues from one another, and regulating intercellular communication. The ECM regulates a cell's dynamic behavior. In addition, it sequesters and acts as a depot for a wide range of cellular growth factors (Robbins and Guo, 2002). Formation of the ECM is essential for several processes such as growth, wound healing, and fibrosis, as metastasis often involves the destruction of the ECM (Liotta et al., 1980).

In addition, we identified 20 abnormal PC-related candidate miRNAs. Among them, hsa-miRNA-26a and eight known PC-related miRNAs were significantly related. hsamiRNA-26a is highly conserved across species and is linked to chromosome 3p22.3/12q14. The fact that increases in hsa-miR-26a were found to be associated with more advanced PC cells suggested a role for miR-26a in the regulation of apoptosis and cell survival, which was substantiated in a report showing increased miR-26a expression in MDA-MB-231 breast cancer cells cultured under hypoxic conditions (Kulshreshtha et al., 2007). Our analysis of miR-26a in these novel cell lines suggested that this is a relevant model to determine miRNA expression patterns associated with PC. Huang et al. (2010) reported that the expression of miRNA-152 (miR-152) was downregulated in livers of HBx transgenic mice in comparison with livers of wild-type mice, based on miRNA microarray and real-time polymerase chain reaction analyses, indicating that miR-152 may play an important role in cancers. A recent study revealed that the essential role of miR-19 was in mediating the oncogenic activity of miR-17-92, and implicated the functional diversity of mir-17-92 components as the molecular basis for its pleiotropic effects during tumorigenesis (Olive et al., 2009). miR-146 was found to be more consistently downregulated in androgen-independent PC cell lines than in androgen-dependent cell lines (Lin et al., 2007). Interestingly, miR-146 was also shown to inhibit the translation of the SDF-1 receptor CXCR4 (Labbaye et al., 2008). The expression 
of miR-30 was inversely related to the amount of connective tissue growth factor (CTGF) in two rodent models of heart disease and in human pathological left ventricular hypertrophy, and overexpression of miR-30 decreased CTGF levels, which was accompanied by decreased collagen production (Duisters et al., 2009). Further analyses will be needed to more closely investigate the individual role of these abnormal PC-related candidate miRNAs in the process of malignant progression in PC.

\section{REFERENCES}

Abrieu A, Brassac T, Galas S, Fisher D, et al. (1998). The Polo-like kinase Plx1 is a component of the MPF amplification loop at the G2/M-phase transition of the cell cycle in Xenopus eggs. J. Cell Sci. 111 (Pt 12): 1751-1757.

Akao Y, Nakagawa Y and Naoe T (2006). MicroRNAs 143 and 145 are possible common onco-microRNAs in human cancers. Oncol. Rep. 16: 845-850.

Baade PD, Youlden DR and Krnjacki LJ (2009). International epidemiology of prostate cancer: geographical distribution and secular trends. Mol. Nutr. Food Res. 53: 171-184.

Clark EA and Brugge JS (1995). Integrins and signal transduction pathways: the road taken. Science 268: 233-239.

Cuddihy AR and O'Connell MJ (2003). Cell-cycle responses to DNA damage in G2. Int. Rev. Cytol. 222: 99-140.

Cummins JM, He Y, Leary RJ, Pagliarini R, et al. (2006). The colorectal microRNAome. Proc. Natl. Acad. Sci. U. S. A. 103: 3687-3692.

Dinasarapu AR, Saunders B, Ozerlat I, Azam K, et al. (2011). Signaling gateway molecule pages - a data model perspective. Bioinformatics 27: 1736-1738.

Djulbegovic M, Beyth RJ, Neuberger MM, Stoffs TL, et al. (2010). Screening for prostate cancer: systematic review and meta-analysis of randomised controlled trials. BMJ 341: c4543.

Duisters RF, Tijsen AJ, Schroen B, Leenders JJ, et al. (2009). miR-133 and miR-30 regulate connective tissue growth factor: implications for a role of microRNAs in myocardial matrix remodeling. Circ. Res. 104: 170-8, 6p.

Golub TR, Slonim DK, Tamayo P, Huard C, et al. (1999). Molecular classification of cancer: class discovery and class prediction by gene expression monitoring. Science 286: 531-537.

He L and Hannon GJ (2004). MicroRNAs: small RNAs with a big role in gene regulation. Nat. Rev. Genet. 5: 522-531.

Hessels D, Klein Gunnewiek JM, van O, I, Karthaus HF, et al. (2003). DD3(PCA3)-based molecular urine analysis for the diagnosis of prostate cancer. Eur. Urol. 44: 8-15.

Huang J, Wang Y, Guo Y and Sun S (2010). Down-regulated microRNA-152 induces aberrant DNA methylation in hepatitis B virus-related hepatocellular carcinoma by targeting DNA methyltransferase 1. Hepatology 52: 60-70.

Iorio MV, Ferracin M, Liu CG, Veronese A, et al. (2005). MicroRNA gene expression deregulation in human breast cancer. Cancer Res. 65: 7065-7070.

Kulshreshtha R, Ferracin M, Wojcik SE, Garzon R, et al. (2007). A microRNA signature of hypoxia. Mol. Cell. Biol. 27: 1859-1867.

Labbaye C, Spinello I, Quaranta MT, Pelosi E, et al. (2008). A three-step pathway comprising PLZF/miR-146a/CXCR4 controls megakaryopoiesis. Nat. Cell Biol. 10: 788-801.

Lee RC, Feinbaum RL and Ambros V (1993). The C. elegans heterochronic gene lin-4 encodes small RNAs with antisense complementarity to lin-14. Cell 75: 843-854.

Lim LP, Lau NC, Garrett-Engele P, Grimson A, et al. (2005). Microarray analysis shows that some microRNAs downregulate large numbers of target mRNAs. Nature 433: 769-773.

Lin SL, Chang D and Ying SY (2007). Hyaluronan stimulates transformation of androgen-independent prostate cancer. Carcinogenesis 28: 310-320.

Liotta LA, Tryggvason K, Garbisa S, Hart I, et al. (1980). Metastatic potential correlates with enzymatic degradation of basement membrane collagen. Nature 284: 67-68.

Marks LS, Fradet Y, Deras IL, Blase A, et al. (2007). PCA3 molecular urine assay for prostate cancer in men undergoing repeat biopsy. Urology 69: 532-535.

Nishizuka Y (1992). Intracellular signaling by hydrolysis of phospholipids and activation of protein kinase C. Science 258: 607-614.

Olive V, Bennett MJ, Walker JC, Ma C, et al. (2009). miR-19 is a key oncogenic component of mir-17-92. Genes Dev. 23: 2839-2849.

Perou CM, Sørlie T, Eisen MB, van de Rijn M, et al. (2000). Molecular portraits of human breast tumours. Nature 406: $747-752$. 
Reinhart BJ, Slack FJ, Basson M, Pasquinelli AE, et al. (2000). The 21-nucleotide let-7 RNA regulates developmental timing in Caenorhabditis elegans. Nature 403: 901-906.

Robbins SL and Guo YW (2002). Pocket Companion to Robbins Pathologic Basis of Disease. Elsevier Health Sciences TW, Philadelphia.

Scheffzek K and Ahmadian MR (2005). GTPase activating proteins: structural and functional insights 18 years after discovery. Cell. Mol. Life Sci. 62: 3014-3038.

Siegel R, Ward E, Brawley O and Jemal A (2011). Cancer statistics, 2011: the impact of eliminating socioeconomic and racial disparities on premature cancer deaths. CA Cancer J. Clin. 61: 212-236.

Taylor BS, Schultz N, Hieronymus H, Gopalan A, et al. (2010). Integrative genomic profiling of human prostate cancer. Cancer Cell 18: 11-22.

Tsang WP and Kwok TT (2008). Let-7a microRNA suppresses therapeutics-induced cancer cell death by targeting caspase-3. Apoptosis 13: 1215-1222.

van 't Veer LJ, Dai H, van de Vijver MJ, He YD, et al. (2002). Gene expression profiling predicts clinical outcome of breast cancer. Nature 415: 530-536.

Yanaihara N, Caplen N, Bowman E, Seike M, et al. (2006). Unique microRNA molecular profiles in lung cancer diagnosis and prognosis. Cancer Cell 9: 189-198. 